\title{
To Establish the Internal Consistency of Inclinometer for Open and Closed Kinematic Chain Measurement of Subtalar Joint
}

\author{
Rohina Kumari ${ }^{1}$, Latha $\mathbf{M}^{2}$ \\ ${ }^{1}$ Lecturer, Department of Prosthetics and Orthotics, Ishwar Institute of Prosthetics and Orthotics, Anna Nagar, \\ Chennai, Tamil Nadu, India \\ ${ }^{2}$ Student, Bachelor of Prosthetics and Orthotics, Ishwar Institute of Prosthetics and Orthotics, Anna Nagar, \\ Chennai, Tamil Nadu, India \\ Corresponding Author: Rohina Kumari
}

DOI: https://doi.org/10.52403/ijhsr.20220222

\begin{abstract}
Introduction: To examine foot and ankle positions, subtalar joint (STJ) measurements are frequently performed in the clinics because of the high prevalence of lower extremity dysfunctions. The STJN position is also used in casting and orthosis fabrication. Unfortunately, current methods of measuring the STJN position are ambiguous or lack the theoretical rigour required for reliable and clinically useful measurements.

Objective: The purpose of this study was to determine if there were any differences in subtalar joint range of motion (ROM) when using OKC and CKC measurement methods, as well as to assess the internal consistency of an inclinometer used in a clinical setting.

Methods: The study included ten healthy subjects. The measurements were taken with the subjects prone (open kinetic chain) and standing (closed kinetic chain). Cronbach's alpha was used to calculate the internal consistency of an inclinometer in the subtalar joint's close and open kinematic chains.

Results: The result indicates that the inclinometer is a good reliable tool for assessing subtalar joint motion in OKC and CKC measurements, as the value for Cronbach's alpha is 0.889 , indicating good reliability.

Discussion \& Conclusion: As a result, it is concluded that this inclinometer will be useful in measuring the open and closed kinematic chain measurements of the subtalar joint. This technique should be viewed as an adjunct to an overall lower extremity biomechanical evaluation. This study provides an optional technique for determining the position or movement of the STJ for clinicians who perform lower extremity biomechanical evaluation.
\end{abstract}

Keywords: Internal consistency, Open kinematic chain, Closed kinematic chain, Inclinometer.

\section{INTRODUCTION}

Objective and reliable measurements of subtalar joint (STJ) motion and position are critical for clinicians involved in the treatment of lower extremity dysfunctions, specifically foot orthotic fabrication, prescription, and management. Overuse injuries to the lower extremity are common, and the position and mobility of the foot and ankle have been shown to influence overuse injuries in the lower extremity. ${ }^{1-4}$

Because of advancements in biomechanics and orthotic management, measurements of subtalar joint neutral are becoming more common. Objective measurements of the rearfoot are required to assist the physical therapist in determining whether the subtalar joint has the range of motion required for walking and running, 
whether there is a biomechanical malalignment at the rearfoot in weightbearing and/or non-weight bearing positions, whether a foot orthotic might correct the biomechanical fault, and, if so, what type of orthotic should be fabricated. A reliable measurement tool will not only allow the physical therapy clinician to make objective, quantifiable recommendations for orthotic management, but it will also allow the clinician to assess a patient's treatment progress. Currently, two methods for measuring subtalar joint neutral have been reported in the literature. The first is a mathematical formula based on measurements of calcaneal inversion and eversion. The second method involves palpating the talus head. There is a lot of controversy about which method is the most accurate and/or reliable. This is an important question because the amount of posting in a foot orthotic is frequently determined by the subtalar joint neutral position in conjunction with lower extremity findings. Non weight bearing measurements of the calcaneus and subtalar joint neutral are highly valued by medical professionals. Therapists have assumed that non weight bearing measurements will provide them with much of the information needed to fabricate a foot orthotic. However, subtalar joint motion normally occurs via a closed kinetic chain, which may not accurately reflect the amount of motion measured in a non weight bearing position. ${ }^{5-7}$

It appears that no studies have been conducted to assess the accuracy of determining STJ neutral position in OKC and CKC. The STJ neutral position is thought to be an important reference position from which motion can be measured. To the best of our knowledge, there are no inclinometer reliability data that support OKC and CKC measurements of the subtalar joint. As a result, the purpose of this study is to determine the internal consistency of an inclinometer in open and closed kinematic measurements of the subtalar joint.

\section{METHODOLOGY}

Ten subjects volunteered to take part in this study (mean age $=27.2$ years, mean height $=165.6 \mathrm{~cm}$, mean weight $=60.5 \mathrm{~kg}$, and mean $\mathrm{BMI}=21.89$ ) table 1 . All subjects denied any neurological or orthopedic pathology affecting their lower extremities. Each subject was informed of the study's purpose and procedure before signing a consent form. All measurements were taken with the same inclinometer on the right lower extremity in both NWB and WB positions by the same person.

Table 1: Descriptive Data of Mean (Age, Height, Weight and BMI)

\begin{tabular}{|c|c|c|}
\hline & $\mathbf{N}$ & Mean \pm S. D \\
\hline Age & 10 & $27.20 \pm 8.09$ \\
\hline Height & 10 & $165.60 \pm 10.94$ \\
\hline Weight & 10 & $60.50 \pm 11.98$ \\
\hline BMI & 10 & $21.89 \pm 2.65$ \\
\hline Valid N(List wise) & 10 & \\
\hline
\end{tabular}

\section{Measurement Procedure}

The subject was placed in a prone position, with the foot and ankle to be measured extended approximately 5 inches off the table and the opposite lower extremity in hip flexion, external rotation, abduction, and knee flexion. A ML calliper is used to find two midpoints on the leg and one on the calcaneus. To create the bisection line, a vertical line was drawn with a straight edge. This line is used to measure the subtalar joints of the OKC and CKC. (Figure 1)

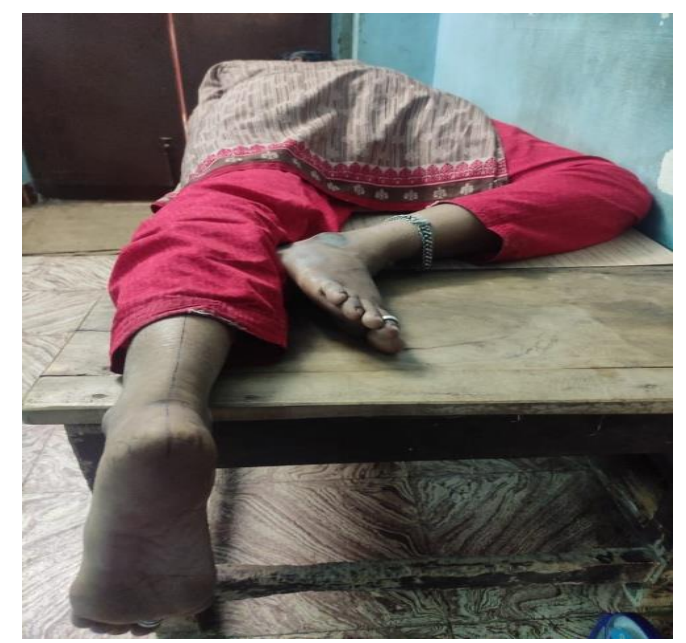

Figure 1. position for taking subtalar joint measurements 
Rohina Kumari et.al. To establish the internal consistency of inclinometer for open and closed kinematic chain measurement of subtalar joint.

\section{Open Kinetic Chain Subtalar Joint Position}

The inclinometer was aligned with the bisection lines of the leg and calcaneus with the axis located between the malleoli using the right hand. After reading and recording the angle, the foot was released and allowed to return to its resting position. The OKC measurement was repeated to establish two values for this measurement. (Figure 2)

\section{Close Kinetic Chain Subtalar Joint Position}

The subjects were asked to stand in bilateral stance position and align the inclinometer in the same manner as for the OKC measurement. The angle was read and recorded. The foot was then released and returned to the resting position. The CKC measurement was repeated to obtain two values. $^{5,8,9}$ (Figure 3 )

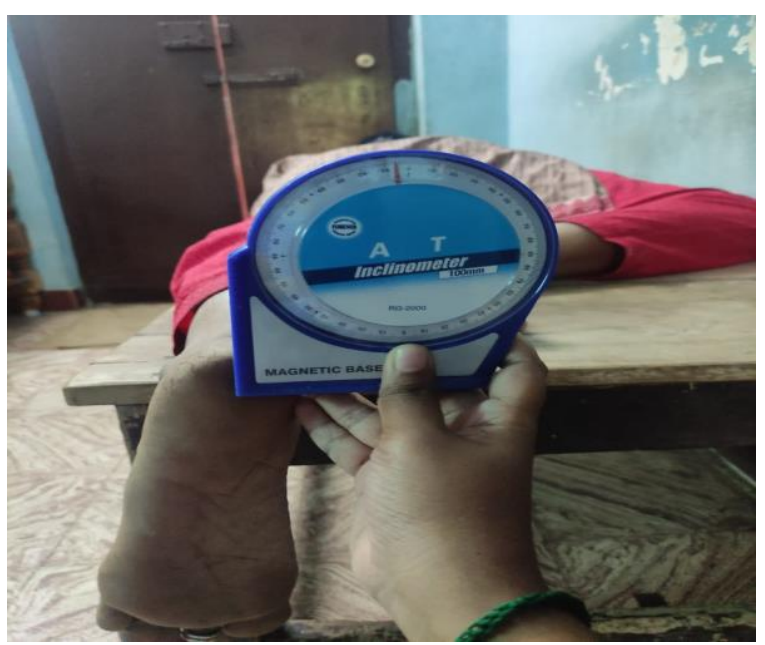

Figure 2. OKC measurement

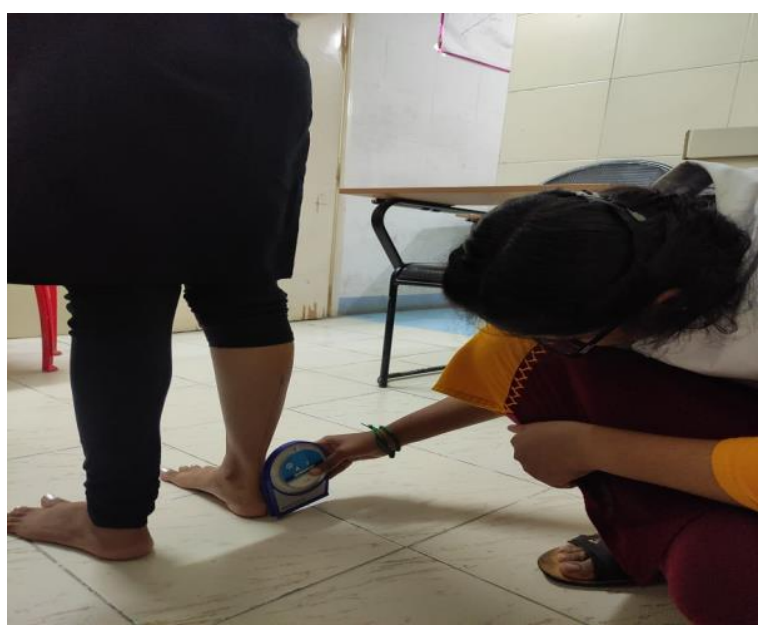

Figure 3. CKC measurement

\section{DATA ANALYSIS}

The data was analysed using the statistical package for social sciences (SPSS) 21 version. Cronbach's alpha was used to calculate the internal consistency of an inclinometer in the subtalar joints closed and open kinematic chains. The significance level was set at $\mathrm{P}<0.05$.

\section{RESULT}

The mean value was calculated by averaging the two values for each measurement. All OKC measurements in this sample of subjects were either zero or inversion position, and all CKC measurements were either zero or eversion position. Cronbach's alpha was used to determine the internal consistency of the inclinometer (table 2)

\begin{tabular}{|l|} 
Table 2 Reliability statistics \\
\begin{tabular}{|l|l|l|}
\hline $\begin{array}{l}\text { Cronbach's } \\
\text { alpha }\end{array}$ & $\begin{array}{l}\text { Cronbach's alpha based on } \\
\text { standardized items }\end{array}$ & $\begin{array}{l}\mathbf{N} \text { of } \\
\text { items }\end{array}$ \\
\hline 0.889 & 0.927 & 2 \\
\hline
\end{tabular}
\end{tabular}

The result indicates that the inclinometer is a good reliable tool for assessing subtalar joint motion in OKC and CKC measurements, as the value for Cronbach's alpha is 0.889 , indicating good reliability. Because the points on the bland Altman plot are scattered all over the place, this suggests that there is no bias in the administration of scale versus another. (Figure 4)

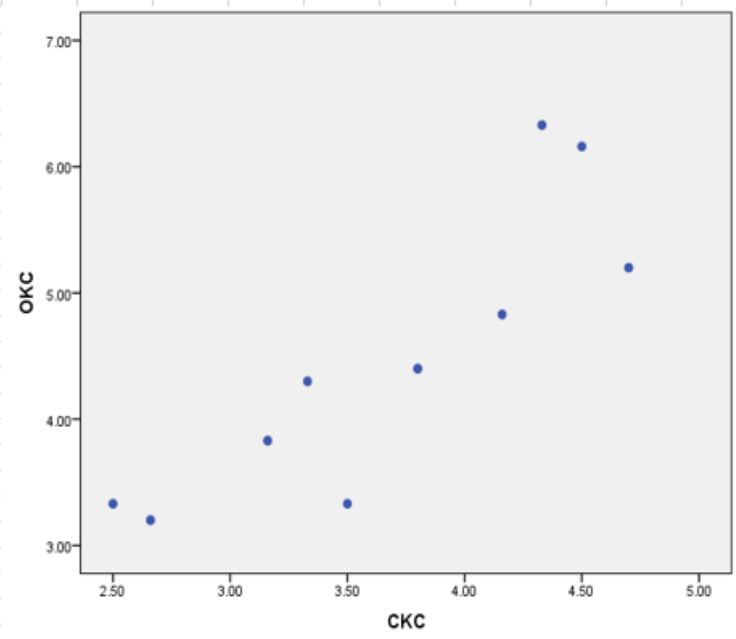

Figure 4. Bland Altman plot 


\section{DISCUSSION}

The result indicates that an inclinometer is a good reliable tool for assessing subtalar joint motion in $\mathrm{OKC}$ and CKC measurements, as the value for Cronbach's alpha is 0.889 , indicating good reliability. This is consistent with the findings of Viitasalo and Kvist, who investigated the reliability of calcaneal inversion and eversion measurements on normal subjects in a non-weight bearing position and discovered a correlation coefficient of $0.84 .^{10}$

Elveru et al. concluded that the goniometric reliability of subtalar and ankle joint measurements in a patient population was satisfactory. For calcaneal inversion and eversion, their ICC values were 0.74 and 0.75 , respectively. ${ }^{11}$

Pandya et al. recently investigated the dependability of goniometric measurements in Duchenne muscular dystrophy patients. Their ICC values for ankle dorsiflexion were 0.90 (intratester) and 0.73 (extratester) (intertester). ${ }^{12}$

Kecia E. Sell et al. also concluded that Intratester results for both resting (ICC $=.85$ ) and neutral positions ( ICC $=.85$ ) were good. The difference between resting and STJ neutral was calculated, and reliability improved to high (ICC $=.91){ }^{1}$

Since humans primarily function in a closed kinematic chain during gait, it is critical that STJ range of motion be measured in the WB position. Although it is well understood that structures and functions differ in NWB versus WB positions, it is also important to measure in NWB positions for patients who present with lower extremity or back dysfunction.

\section{CONCLUSION}

Clinical measurements of the STJN position, as used in this study, can be reliable when performed by the same examiner over a short period of time. Our findings suggest that subtalar joint neutral can be accurately determined in the weightbearing position. The inclinometer should help the clinician to assess the STJ position and, more importantly, subtalar joint mobility more accurately. This method, we believe, is easier to implement. In theory, error should be reduced through proper training as well as taking multiple measurements and calculating the mean of these measurements. Furthermore, additional research should be conducted to investigate the relationship of this technique to determining orthotic prescription, the efficacy of orthotic treatment, and the reliability for patients with actual pathologies.

\section{Acknowledgement: None}

\section{Conflict of Interest: None}

\section{Source of Funding: None}

\section{Ethical Approval: Approved}

\section{REFERENCES}

1. Sell KE, Verity TM, Worrell TW, Pease BJ, Wigglesworth J. Two measurement techniques for assessing subtalar joint position: A reliability study. Journal of Orthopaedic and Sports Physical Therapy. 1994;19(3):162-7.

2. Ator R, Gunn K, McPoil TG, Knecht HG. The Effect of Adhesive Strapping on Medial Longitudinal Arch Support before and after Exercise. J Orthop Sports Phys Ther. 1991; 14(1):18-23. doi: 10.2519/jospt.1991. 14.1.18. PMID: 18796830.

3. Brody DM. Techniques in the evaluation and treatment of the injured runner. Orthop Clin North Am. 1982;13(3):541-58.

4. Donatelli R, Hurlbert C, Conaway PD, Pierre RST. Biomechanical Foot Orthotics :

A Retrospective Study. Journal of Orthopaedic \& Sports Physical Therapy. 1988;10(6):205-11.

5. Smith-Oricchio K, Harris BA. Interrater reliability of subtalar neutral, calcaneal inversion and eversion. Journal of Orthopaedic and Sports Physical Therapy. 1990;12(1):10-5.

6. McPoil Jr BR. The foot and ankle: Biomechanical evaluation and treatment. orthopaedics and sports physical therapy. $1985 ; 2: 313-25$. 
7. MJ W. podiatric physical therapy. clinical education associates. 1987;

8. Picciano AM, Rowlands MS, Worrell T. Reliability of Open and Closed Kinetic Chain Subtalar Joint Neutral Positions and Navicular Drop Test. Journal of Orthopaedic \& Sports Physical Therapy. 1993;18(4):553-8.

9. Elveru RA, Rothstein JM, Lamb RL, Riddle DANL. Methods for Taking Subtalar Joint Measurements A Clinical Report. Physical Therapy. 1988;68(5):678-82.

10. Viitasalo JT. Some biomechanical aspects of the foot and ankle in athletes with and without shin splints. the american journal of sports medicine. 1983;11(3):125-30.
11. Elveru RA, Rothstein JM, Lamb RL. Goniometric Reliability in a Clinical Setting Subtalar and Ankle Joint Measurements. Physical Therapy. 1988;68(5):672-7.

12. Pandya S, Florence JM, King WM, Robison JD, Oxman M, Province MA. Reliability of Goniometric Measurements in Patients with Duchenne Muscular Dystrophy. Physical Therapy. 1985;65(9):1339-41.

How to cite this article: Kumari R, Latha M. To establish the internal consistency of inclinometer for open and closed kinematic chain measurement of subtalar joint. Int $J$ Health Sci Res. 2022; 12(2):158-162. DOI: https://doi.org/10.52403/ijhsr.20220222 